VIEWPOINT

Andrew S. Levey, MD Division of Nephrology, Tufts Medical Center, Boston, Massachusetts.

Lesley A. Inker, MD, MS Division of Nephrology, Tufts Medical Center, Boston, Massachusetts.

Josef Coresh, MD, PhD Departments of Epidemiology. Biostatistics, and Medicine, Johns Hopkins University, Baltimore, Maryland.

$\leftarrow$

Counter Viewpoint page 559

\section{Corresponding} Author: Andrew S. Levey, MD, Tufts Medical Center. Box 391, 850 Washington St, Boston, MA 02111 (alevey @tuftsmedicalcenter .org).

\title{
Chronic Kidney Disease in Older People
}

\section{Should current guidelines be changed to require age calibration for diagnosis and classification of chronic kidney disease? -No.}

Chronic kidney disease (CKD) is a global public health problem, affecting more than $10 \%$ of the world's population and more than half of adults older than 70 years. ${ }^{1}$ Chronic kidney disease is one of several conditions that are common in older people, such as hypertension, diabetes, and hyperlipidemia, and are associated with an increased risk of cardiovascular disease. Like CKD, the criteria for defining and classifying these vascular disease risk factors do not vary by age, although age is important for assessing prognosis and determining treatment. The solution to the public health problem of CKD is to improve strategies for prevention and treatment and tailor these strategies to each patient's age and risk, rather than redefine CKD.

\section{Current Guidelines Are Widely Accepted} The 2012 KDIGO (Kidney Disease: Improving Global Outcomes) CKD guideline represents a mature, broadbased consensus for clinical practice, research, and public health. Based on review of data on prognosis, KDIGO recommended no change to the definition of CKD, as have all the other major workgroups since the definition was introduced in 2002. ${ }^{2}$ Specifically, KDIGO retained the criteria for the definition of CKD without change: glomerular filtration rate (GFR) less than $60 \mathrm{~mL} / \mathrm{min} / 1.73 \mathrm{~m}^{2}$ or markers of kidney damage (including albuminuria $>30 \mathrm{mg} / \mathrm{d}$ ) for more than 3 months. The classification retained staging by GFR but added staging by albuminuria and cause of disease (referred to as CGA staging) and added recommendations to assess the prognosis of CKD using risk prediction instruments. ${ }^{3}$ Glomerular filtration rate and albuminuria are continuous variables, but categorization is required to facilitate clinical recommendations.

Because early stages of CKD are generally asymptomatic, KDIGO recommended laboratory testing to detect CKD in populations at increased risk. KDIGO recommended the CKD-EPI (Chronic Kidney Disease Epidemiology Collaboration) equations as the preferred methods for estimating GFR from serum creatinine, cystatin C, or both and recommended estimating albuminuria from albumin-to-creatinine ratio in untimed "spot" urine samples. ${ }^{4}$ The accuracy of these methods does not vary substantially by age in adults.

\section{CKD Is Common in Older People}

Many studies show that mean GFR is lower and albuminuria is higher in older people. Because these abnormalities are common, some have considered them to be the consequence of "normal aging," but there is considerable evidence that they are related to factors beyond aging. First, there is wide variation in the levels of GFR and albuminuria in older people, with some people exhibiting virtually normal levels and others having severe abnormalities. Second, lower GFR and higher albuminuria are associated with other abnormalities in kidney function and structure, such as decreased renal plasma flow, reduced maximal urine concentration and acidification, glomerular and arterial sclerosis, and tubular atrophy, which are considered pathological when observed in younger people. Third, the kidney is a highly vascular organ, and levels of GFR and albuminuria are related to the severity of vascular disease risk factors and vascular disease, suggesting that these abnormalities may be the consequence of vascular disease in the kidney. Therefore, an attempt to understand the cause of lower GFR and higher albuminuria in aging is more appropriate than considering it "normal" because it is common.

\section{CKD Is Associated With Increased Risk in Older People}

Decreased GFR and increased albuminuria are associated with increased risk at all ages, but risk associations differ in older vs younger people. A meta-analysis including 1885185 participants from 33 general population and high-risk cohorts demonstrated that relative risk is lower and excess risk is higher at older age vs younger age for mortality due to decreased estimated GFR (eGFR) or increased urine albumin/creatinine ratio. ${ }^{5}$ Similar findings were observed for risks of mortality and kidney failure in 38612 participants from 13 CKD cohorts. In general, the pattern of lower relative risk and higher excess risk in older vs younger age is observed for common vascular disease risk factors. ${ }^{6}$ Possibly, the explanation relates to the multiplicity of vascular risk factors at older age, which makes for a higher absolute risk and lower relative risk for each factor. Demonstration of excess risk associated with decreased GFR and increased albuminuria in older people indicates that these abnormalities should not be dismissed as "normal."

\section{What Issues Are Important to Patients?}

There is debate about overdiagnosis of common chronic conditions in older people, contributing to "medicalization of aging," vs underdiagnosis, leading to missed opportunities for disease detection and prevention and treatment of adverse outcomes. However, this is a debate for physicians and public health officials, not for patients.

Patients would like to know the cause of their condition, whether it is called a "kidney disease" or "decreased kidney function." Irrespective of the term used to describe the condition, an evaluation is required to explain the cause.

Patients would like to have treatment options to address the cause of their disease, slow its progression, and 
prevent complications. There are many examples of older people benefiting from treatment of common conditions that are often labeled as an inevitable part of aging. Few established therapies for kidney disease are available at the current time, and previous trials have not assessed all the outcomes that are important to patients. ${ }^{7}$ The need for additional treatments to improve outcomes for CKD should be a call for action as opposed to inaction.

Patients value information about their health. The diagnosis of a chronic health condition has important implications that clinicians can help patients address. It would be inappropriate for a clinician to fail to disclose the true state of a patient's health.

\section{An Age-Calibrated Definition Will Not Work}

Age calibration does not solve the problem of needing to categorize continuous values of GFR and albuminuria for disease definition and classification. Indeed, age calibration will require even more categories based on the combination of age, GFR, and albuminuria.

Age calibration is too complicated and would require another term for decreased GFR or increased albuminuria that is not normal but not sufficient for the diagnosis of CKD. In the past, the terms "renal impairment" or "renal insufficiency" were used without definition, leading to the state of "chronic renal confusion." Age calibration could create the possibility that a patient's disease classification could change because of age without a change in health status. For example, a patient with childhood CKD, for example, vesicoureteral reflux or IgA nephropathy, could "improve" as the patient ages, even though there is no change in GFR and albuminuria.

Age calibration does not change major treatment recommendations: they are based on the level of GFR and albuminuria, rather than the diagnosis of CKD. Examples include drug dosing of antibiotics or chemotherapy and contraindications to toxic agents such as nonsteroidal anti-inflammatory drugs and radiographic contrast based on GFR, and antihypertensive therapy with angiotensinconverting enzyme inhibitors and angiotensin II receptor blockers based on albuminuria.

Age calibration is not well suited to address different risk relationships for different outcomes and covariate patterns in indi- vidual patients. Risk assessment tools are preferred for this purpose. In addition, age calibration does not address the issues that are important to patients.

\section{What Should Be Done: Full Disclosure}

The 2012 KDIGO definition and CGA staging of CKD should be adopted and disseminated to increase awareness of CKD among clinicians, patients, and the public. Detection, evaluation, and management should be individualized based on the patient's risk. Patients at increased risk should undergo testing for CKD. Estimated GFR based on serum creatinine (eGFRcr) and urinalysis are often available from routine health encounters for acute and chronic illness. Other markers of kidney damage can be assessed among patients with a clinical suspicion of CKD. When confirmation of decreased eGFR is needed, eGFR can be computed from creatinine and standardized cystatin C (eGFRcr-cys) or GFR can be measured. ${ }^{4}$ Patients with CKD should be evaluated for the cause of disease and the presence of complications, with the extent of the evaluation based on risk of adverse outcomes and the life expectancy of the patient. In general, young people are at greater risk of progression to kidney failure, whereas older people are at greater risk of other complications and death. ${ }^{3}$ The "kidney failure risk equation," which incorporates age, GFR, and urine albumin/ creatinine ratio, can be used to quantify risk of kidney disease progression requiring treatment with dialysis and transplantation ${ }^{9}$; other instruments can be used to quantify risk for death without treatment for kidney failure. ${ }^{10}$ Patients at high risk can undergo more intensive evaluation and management tailored to reversible risk factors for specific outcomes.

It is time to move forward from debate about the validity of the data about risk and the semantics of the definition and classification of CKD, to the challenges of seeking the explanation for why CKD is so common, testing strategies for its prevention and treatment, and answering questions that are important to patients. Physicians should fully disclose the risks of CKD and opportunities for treatment to patients of all ages and evaluate and manage cases according to the level of risk. Communicating risks to patients is a challenging task, but one physicians should accept rather than avoid.
ARTICLE INFORMATION

Published Online: May 29, 2015.

doi:10.1001/jama.2015.6753.

Conflict of Interest Disclosures: All authors have completed and submitted the ICMJE Form for Disclosure of Potential Conflicts of Interest. Dr Levey reports that he was chair of the workgroup for the 2002 KDOQI (Kidney Disease Outcomes Quality Initiative) CKD guideline and member of the workgroup for the 2012 KDIGO CKD guidelines. $\mathrm{Dr}$ Inker reports that she was co-chair of the workgroup for the $2013 \mathrm{KDOQI}$ commentary on the 2012 KDIGO CKD guideline. Dr Levey reports being the principal investigator and $\mathrm{Dr}$ Inker reports being the clinical director for the CKD-EPI (Chronic Kidney Disease Epidemiology Collaboration) research group, which developed the CKD-EPI equations for glomerular filtration rate (GFR) estimation. Drs Levey, Inker, and Coresh have applied for a patent for precise estimation of GFR using a panel of filtration markers. No other disclosures were reported.
Additional Contributions: We thank Aghogho Okparavero, MD, Tufts Medical Center, for assistance in manuscript preparation. He was not compensated for his contribution.

\section{REFERENCES}

1. Levey AS, Coresh J. Chronic kidney disease. Lancet. 2012;379(9811):165-180.

2. Kidney Disease: Improving Global Outcomes (KDIGO). KDIGO 2012 clinical practice guideline for the evaluation and management of chronic kidney disease. Kidney Int Suppl. 2013;3(1):1-150.

3. Tangri N, Kitsios GD, Inker LA, et al. Risk prediction models for patients with chronic kidney disease. Ann Intern Med. 2013;158(8):596-603.

4. Levey AS, Becker C, Inker LA. Glomerular filtration rate and albuminuria for detection and staging of acute and chronic kidney disease in adults. JAMA. 2015;313(8):837-846.

5. Hallan SI, Matsushita K, Sang Y, et al; Chronic Kidney Disease Prognosis Consortium. Age and association of kidney measures with mortality and end-stage renal disease. JAMA. 2012;308(22): 2349-2360.

6. Labarthe DR. Epidemiology and Prevention of Cardiovascular Disease: A Global Challenge. 2nd ed. Sudbury, MA: Jones \& Bartlett; 2009.

7. Uhlig K, Levey AS. Developing guidelines for chronic kidney disease. Ann Intern Med. 2012;156 (8):599-601.

8. Hsu CY, Chertow GM. Chronic renal confusion: insufficiency, failure, dysfunction, or disease. Am J Kidney Dis. 2000;36(2):415-418.

9. Tangri N, Stevens LA, Griffith J, et al. A predictive model for progression of chronic kidney disease to kidney failure. JAMA. 2011;305(15):15531559.

10. Bansal N, Katz R, De Boer IH, et al. Development and validation of a model to predict 5-year risk of death without ESRD among older adults with CKD. Clin J Am Soc Nephrol. 2015;10(3): 363-371. 